\title{
mrSNP: Software to detect SNP effects on microRNA binding
}

\author{
Mehmet Deveci ${ }^{1 *}$, Ümit V Çatalyürek ${ }^{2}$ and Amanda Ewart Toland ${ }^{3}$
}

\begin{abstract}
Background: MicroRNAs (miRNAs) are short (19-23 nucleotides) non-coding RNAs that bind to sites in the $3^{\prime}$ untranslated regions ( $3^{\prime} \cup T R$ ) of a targeted messenger RNA (mRNA). Binding leads to degradation of the transcript or blocked translation resulting in decreased expression of the targeted gene. Single nucleotide polymorphisms (SNPs) have been found in $3^{\prime} U T R s$ that disrupt normal miRNA binding or introduce new binding sites and some of these have been associated with disease pathogenesis. This raises the importance of detecting miRNA targets and predicting the possible effects of SNPs on binding sites. In the last decade a number of studies have been conducted to predict the location of miRNA binding sites. However, there have been fewer algorithms published to analyze the effects of SNPs on miRNA binding. Moreover, the existing software has some shortcomings including the requirement for significant manual labor when working with huge lists of SNPs and that algorithms work only for SNPs present in databases such as dbSNP. These limitations become problematic as next-generation sequencing is leading to large numbers of novel variants in 3'UTRs.
\end{abstract}

Result: In order to overcome these issues, we developed a web-server named mrSNP which predicts the impact of a SNP in a $3^{\prime} U T R$ on miRNA binding. The proposed tool reduces the manual labor requirements and allows users to input any SNP that has been identified by any SNP-calling program. In testing the performance of mrSNP on SNPS experimentally validated to affect miRNA binding, mrSNP correctly identified 69\% $(11 / 16)$ of the SNPs disrupting binding.

Conclusions: mrSNP is a highly adaptable and performing tool for predicting the effect a $3^{\prime} U T R$ SNP will have on miRNA binding. This tool has advantages over existing algorithms because it can assess the effect of novel SNPs on miRNA binding without requiring significant hands on time.

Keywords: miRNA, SNP, mRNA, microRNA binding

\section{Background}

MicroRNAs (miRNAs) are predicted to regulate over 60\% of all genes and as such have a significant impact on cell function and biology [1]. MiRNAs bind to the 3'UTR of an mRNA which results in decreased expression of the targeted gene. Thus, miRNA binding analysis is essential for any biological workflow that examines gene expression.

Processing of miRNAs is a multi-step process. First the miRNA transcript folds into a hairpin loop which is called the pri-miRNA. The hairpin loop is processed further into a pre-miRNA and is exported to the nucleus where it

\footnotetext{
*Correspondence: mdeveci@bmi.osu.edu

1 Biomedical Informatics, Computer Science and Engineering, The Ohio State University, Columbus, Ohio, USA

Full list of author information is available at the end of the article
}

binds with dicer and is processed into a mature miRNA of roughly 19-23 nucleotides in length. The mature miRNA together with the protein-silencing complex (RISC) seeks and binds to mRNA at target sites. Binding can cause mRNA destabilization leading to translational repression or direct degradation of the mRNA target. Initially, miRNA targets were detected through classical genetic techniques. Due to the painstaking nature of these experiments and the lack of high-throughput protocols, there is a great need to develop computational techniques to determine miRNA targets. After it was shown that 3'UTR regions contain binding sites for miRNAs that have some degree of complementarity, various methods of computational predictions were developed. 
Generally, plant miRNAs have perfect base-pairing with their target, causing its degradation. In animals, miRNAs can also form limited base-pairing, primarily between the 2nd and 7th bp from the 5' end of miRNA (seed of miRNA), which leads to translational repression. This imprecise sequence matching makes it more difficult to predict miRNA targets in animals with high accuracy. Different techniques have been proposed to predict mammalian miRNA-mRNA binding. These include the pattern of base pairing, thermodynamic stability of the miRNAmRNA hybrid, comparative sequence analysis for conservation, and examination of multiple target sites [2].

Several software programs have been developed that utilize one or more of these methods to identify miRNA binding sites in the genome. TargetScan checks thermodynamic stability and conservation of the target sites in related species [3]. Miranda combines the pattern of base pairing, the thermodynamic stability of the miRNAmRNA hybrid and comparative sequence analysis for conservation [4]. RNAhybrid determines the optimal and subobtimal binding energies between a given miRNA and its mRNA target [5]. MicroInspector detects binding sites according to complementarity using two sliding windows of 6 nucleotides in length [6] . Pictar requires base-pair matches in the seed region of miRNA, applies filtering by calculating thermodynamic binding energy, and assigns a likelihood score using a Hidden Markov Model for each binding [7]. Diana-microT considers principles of binding energy and conservation $[8,9]$. It also integrates biological pathways and analysis of interactions between predicted target genes.

Disease-associated functional SNPs may alter gene expression. Therefore, the relationship between SNPs and miRNAs becomes important for understanding the role of SNPs on disease [10]. Although there are many miRNA binding prediction tools that have been studied in the last decade, fewer studies to assess SNP effects on miRNA binding have been published [11-15]. Recently, the databases microSNiPer, Patrocles, Mirsnpscore, miRdSNP, MirSNP, PolymiRTS have been released [16-21]. These databases follow similar algorithms as those utilized by the miRNA prediction tools in order to detect the effects of the SNPs on miRNA binding. These algorithms are run on the whole genome for all SNPs present in a genomic database like dbSNP, then results are stored. Users can query the results using SNP, gene or miRNA IDs. One of the deficiencies of these databases is that they only work for SNPs that already exist in databases and do not work for novel or unreported SNPs. Moreover, if the list of SNPs is large, the web interface of the tools may require an infeasible amount of manual labor. With the advent of next-generation sequencing technologies such as RNA-Seq, exome and whole genome sequencing, thousands of novel SNPs in 3'UTRs are being identified.
RNA-Seq, which sequences all expressed genes in a sample, provides concordant gene expression and SNP data. Since a substantial number of the detected SNPs are previously undocumented, use of algorithms that require a SNP to be present in dbSNP may not meet the needs of researchers using RNA-Seq or other next-generation sequencing methods. Currently, when a novel SNP is encountered, a user can compare the location of the SNP against the predicted and validated miRNA target sites using the current prediction tools which is fairly labor intensive. The probability of the SNP disturbing a binding site can be considered to be proportional to the distance of the SNP to the seed of the target site. However, a SNP may not affect binding even when it is very close to the miRNA target seed region. Moreover, a SNP may introduce a totally new binding with a new miRNA, which is impossible to capture with the current databases. Thus, next-generation sequencing data demands new computational tools to relate the SNP and gene expression, which motivated us to develop a web-based tool, named mrSNP, to overcome the shortcomings of existing tools.

\section{Implementation}

The implementation of the mrSNP is presented in Figure 1. All the 3' UTR sequences and phastCons scores of the each nucleotides are downloaded from the UCSC Database using the Genome Table Browser [22]. Each chromosome is stored in a single file, where each sequence has information including gene name and 3'UTR sequence coordinates. All available miRNAs are downloaded from the mirBase database and clustered according to their conservation across species using the information obtained from the TargetScan prediction tool $[3,23]$. The software accepts input SNPs with the related information containing the organism, the assembly according to which the mapping is done, the chromosome on which the SNP is located, the position of the SNP in the given chromosome, and the SNP alleles. Once this information is provided, the software searches for the sequence where it is located. If SNP is not located in a denoted 3' UTR sequence, no further calculation is done and the software reports the SNP as, "not in 3'UTR". If the SNP is found in a 3'UTR, the 79 basepairs (bp) of sequence that contains the SNP at the center is returned at this step. This length (79bp) was chosen based on the observation that the typical maximum size of an miRNA is $25 \mathrm{bp}$ and a maximum $15 \mathrm{bp}$ loop is allowed in the binding. Therefore, we allow a miRNA binding site to have a maximum length of $40 \mathrm{bp}$. If a SNP is to affect miRNA binding, it will be located in the miRNA's binding site whose start/end nucleotide can be at most $39 \mathrm{bp}$ apart from the SNP. Therefore, a 79 bp sequence (40 bp + $39 \mathrm{bp}$ ) is the minimal sequence to use for calculating potential miRNA binding differences. Once this sequence 


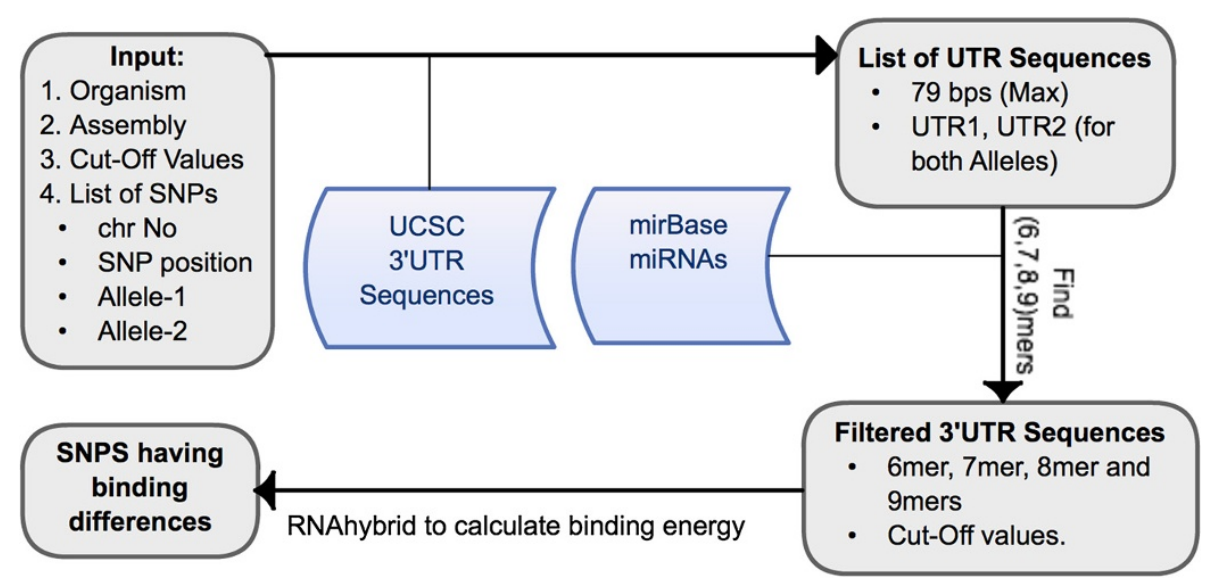

Figure 1 The workflow of mrSNP software.

is obtained, it is duplicated and each SNP allele is substituted in the correct position. After this point, for each miRNA stored we check the existence of a minimum of 6 consecutive Watson-Crick (W-C) matches starting from second position of the miRNA seed region.

The remainder of the approach is adapted from [9]. A sequence with $6(7,8$, or 9) consecutive matches is called a 6 mer (7mer etc.). We allow a single G:U wobble for 7,8 and 9 mer sequences. If no instances satisfy matching criteria, the miRNA and the sequence couple are not investigated further, and we conclude that the miRNA does not bind to this region. Moreover, if the sequence has at least 7 Watson-Crick matches in the seed region, it is considered as a miRNA binding site immediately. For weaker bindings such as the 6mers, or 7, 8 and 9mer sequences containing a single G-U wobble, we calculate the binding energy with RNAhybrid [5]. RNAhybrid runs a dynamic programming algorithm that finds the suboptimal binding energy between 2 sequences. For 6 mers and 7 mers ( 8 mers and 9 mers), we say that microRNA binds to a sequence if its binding energy is higher than $74 \%$ (60\%) of the maximum binding energy. The numbers and methods used are adapted from [9]. For a given SNP-miRNA couple, the steps explained above are followed for both of the SNP sequences. If one of the them satisfies the binding criteria, while the other does not, we report this as a binding difference.

In the literature, many of the prediction tools apply a post-processing step to reduce the false positive rate of the binding predictions. This is performed using the conservation of the target site across different species. If the target site is conserved over different species, the binding possibility is considered to be higher. Although mrSNP does not filter out the results with this post-processing method, it calculates the conservation score (CS) of the seed region using the phastCons scores provided by UCSC database. For each prediction, CS is obtained as the average phastCons score of the nucleotides in the seed region. Then, it reports the probabilistic CS of the seed region as well as the conservation of the miRNA over the species.

\section{Usage}

mrSNP software is publicly available from http://mrsnp. osu.edu. First, the user selects the organism and the assembly used in the analysis. mrSNP currently supports 11 organisms with the available assemblies.

Once organism and assembly are chosen, the user inputs the list of SNPs by either typing in the textbox or uploading a file. Each line should contain a single SNP with chromosome number, SNP position, and first and second allele. Each entry is separated by a space. An e-mail address can be provided for obtaining results. Also, the cut-off ratios to apply 6, 7, 8 and 9mers are parametrized for the option of using different ratios for different organisms. When a job is submitted, the user is directed to another page summarizing inputs and a link to the results page. Once the result is ready, it is displayed in a table containing the fields: chromosome, SNP position, target gene, the binding miRNA, the binding energy difference, the binding energies of each SNP, the cut-offs applied to each sequence, and the alignment of the bindings. If a SNP is not located in any 3'UTR region, or if it does not affect any miRNA bindings, the related information is reported at target gene field. A downloadable file is also provided. If there are any errors found, a link to the error page is given at the bottom of the page.

\section{Results and discussion}

Although mrSNP does not require its input to be validated SNPs, in order to evaluate the accuracy of mrSNP, we ran a series of experiments on multiple sets of experi- 
mentally validated SNP-miRNA couples for human hg19 assembly.

In the first set of validation experiments, we ran experimentally validated disease associated SNPs used in the experiments of [20] in order to compare mrSNP with different databases. 16 SNPs

which are both associated with disease and experimentally validated to disrupt miRNA binding were chosen. Table 1 gives the results of this experiment. For each
SNP-miRNA pair, the table reports the SNP's rsID, the name of the miRNA, the location of the SNP, SNP alleles, the success/failure of mrSNP, and the explanation of the behavior. In this experiment, $\mathrm{mrSNP}$ is able to recover 11 disease associated SNPs out of 16. Among the 5 SNPs which are predicted not to affect miRNA bindings, the effect of $r s 13212041$ on hsa-miR-96 is not captured as the SNP is not located in the 3'UTR. The effects of 3 SNPs (rs2735383, rs34764978, rs9341070) are

Table 1 Results of mrSNP on 16 experimentally validated disease-associated SNPs described in [20]

\begin{tabular}{|c|c|c|c|c|c|c|c|}
\hline \multirow{2}{*}{$\frac{\text { SNP }}{\text { rs1063320 }}$} & \multirow{2}{*}{$\begin{array}{l}\text { miRNA } \\
\text { hsa-miR-152 }\end{array}$} & \multirow{2}{*}{$\frac{\text { Chr }}{6}$} & \multirow{2}{*}{$\begin{array}{r}\text { Position } \\
29798749\end{array}$} & \multicolumn{2}{|c|}{ Alleles } & \multirow{2}{*}{$\begin{array}{c}\text { Success } \\
\checkmark\end{array}$} & \multirow{2}{*}{$\begin{array}{l}\text { Explanation } \\
\text { Binds one of the sequences with } 7 \text { consecutive matches. } \\
\text { SNP breaks the match on the } 7 \text { th position, min. match } \\
\text { critera is not satisfied. }\end{array}$} \\
\hline & & & & C & G & & \\
\hline rs1063320 & hsa-miR-148a & 6 & 29798749 & C & G & $\checkmark$ & $\begin{array}{l}\text { Binds one of the sequences with } 8 \text { consecutive matches. } \\
\text { SNP breaks the match on the } 7 \text { th position, min. match } \\
\text { critera is not satisfied. }\end{array}$ \\
\hline rs1063320 & hsa-miR-148b & 6 & 29798749 & $C$ & $G$ & $\checkmark$ & $\begin{array}{l}\text { Binds one of the sequences with } 7 \text { consecutive matches. } \\
\text { SNP breaks the match on the } 7 \text { th position, min. match } \\
\text { critera is not satisfied. }\end{array}$ \\
\hline rs3134615 & hsa-miR-1827 & 1 & 40362066 & A & $C$ & $\checkmark$ & $\begin{array}{l}\text { Binds one of the sequences with } 8 \text { consecutive matches. } \\
\text { SNP breaks the match on the } 6 \text { th position, min. match } \\
\text { critera is not satisfied. }\end{array}$ \\
\hline rs4245739 & hsa-miR-191 & 1 & 204518842 & A & $C$ & $\checkmark$ & $\begin{array}{l}\text { Binds one of the sequences with } 8 \text { consecutive matches. } \\
\text { SNP breaks the match on the } 5 \text { th position, min. match } \\
\text { critera is not satisfied. }\end{array}$ \\
\hline rs56109847 & hsa-miR-510 & 3 & 183824557 & A & G & $\checkmark$ & $\begin{array}{l}\text { Binds one of the sequences with } 8 \text { consecutive matches. } \\
\text { SNP breaks the match on } 5 \text { th position, min. match critera } \\
\text { is not satisfied. }\end{array}$ \\
\hline rs5186 & has-miR-155 & 3 & 148459988 & $A$ & $C$ & $\checkmark$ & $\begin{array}{l}\text { Binds one of the sequences with } 7 \text { consecutive matches. } \\
\text { SNP breaks the match on 4th position, min. match critera } \\
\text { is not satisfied. }\end{array}$ \\
\hline rs 1434536 & hsa-miR-125b & 4 & 96075965 & $T$ & $C$ & $\checkmark$ & $\begin{array}{l}\text { Binds one of the sequences with } 8 \text { consecutive matches. } \\
\text { SNP introduces a GU wobble on 8th position (7th of seed), } \\
\text { the binding energy is below the cut off. }\end{array}$ \\
\hline rs193302862 & hsa-miR-24 & 13 & 84452863 & $C$ & $T$ & $\checkmark$ & $\begin{array}{l}\text { Binds one of the sequences with } 9 \text { consecutive matches. } \\
\text { SNP introduces a GU wobble on 8th position (7th of the } \\
\text { seed), the binding energy is lower than cut off. }\end{array}$ \\
\hline rs8126 & hsa-miR-184 & 14 & 103603569 & $C$ & $T$ & $\checkmark$ & $\begin{array}{l}\text { Binds one of the sequences with } 7 \text { consecutive matches. } \\
\text { SNP introduces a GU wobble on 6th position (7mer with a } \\
\text { GU wobble), the binding energy is below the cut off. }\end{array}$ \\
\hline rs12720208 & has-miR-433 & 8 & 16850399 & G & A & $\checkmark$ & $\begin{array}{l}\text { Binds one of the sequences with } 7 \text { consecutive matches. } \\
\text { SNP introduces a GU wobble on 6th position, the binding } \\
\text { energy is lower than cut off. }\end{array}$ \\
\hline rs13212041 & hsa-miR-96 & 6 & 78171124 & C & $\mathrm{T}$ & $x$ & SNP is not in $3^{\prime} U T R$ \\
\hline rs2735383 & hsa-miR-629 & 8 & 90947269 & C & G & $x$ & $\begin{array}{l}\text { miRNA is predicted not to bind either of the sequences. } \\
\text { There exists a mistmatch on the } 4 \text { th position regardless of } \\
\text { the SNP. SNP further breaks the match on 3rd position. }\end{array}$ \\
\hline rs34764978 & has-miR-24 & 5 & 79924683 & A & G & $x$ & $\begin{array}{l}\text { miRNA is predicted not to bind either of the sequences. } \\
\text { Both have only } 6 \text { matches with GU wobble. }\end{array}$ \\
\hline rs9341070 & has-miR-206 & 6 & 152420197 & C & $\mathrm{T}$ & $x$ & $\begin{array}{l}\text { miRNA is predicted not to bind either of the sequences. } \\
\text { min. match criteria cannot be satisfied in both }\end{array}$ \\
\hline rs67384697 & hsa-miR-148a & 6 & 31236683 & $C$ & - & $x$ & $\begin{array}{l}\text { miRNA is predicted to bind both of the sequences. SNP } \\
\text { reduces } 11 \text { consecutive match to } 9 \text {. }\end{array}$ \\
\hline
\end{tabular}


not recovered as the sequences for both of the alleles do not satisfy the minimum matching criteria. On the other hand, although mrSNP recognizes the binding energy change of hsa-miR-148a for rs67384697; both of the sequences satisfy the matching criteria, and no effect on miRNA binding is detected. Among the 11 SNP-miRNA pairs that are successfully detected by mrSNP, 7 of them are captured because the SNPs break a matching in the seed region which causes the sequence not to meet the minimum matching criteria. The other 4 SNPs introduce GU wobbles in the seed region. The binding of these SNPs are predicted to be disrupted since the binding energies are calculated to be lower than the required threshold. In comparing the results of mrSNP to other databases and algorithms described in [20], MirSNP, PolymiRTS, Mirsnpscore, and Patrocles are able to capture 12, 7, 7, and 5 of the disease-associated SNPs respectively. Thus, mrSNP outperforms all tools except MirSNP. MirSNP detected similar binding differences as mrSNP with the exception of capturing the rs67384697 $h s a-m i R-148 a-3 p$ pair. MirSNP reports this pair as "Enhance/Decrease" which means a binding energy difference between two sequences for each the allele of the SNP was measured, rather than a break in the binding. As explained in Table 1, a binding energy difference between the alleles is also captured by mrSNP, however, it is not reported because both of them satisfy the matching criteria.

In the next set of validation experiments, we tested mrSNP on SNPs obtained from the miRdSNP database [19]. We chose the SNPs that map to the miRNA targets predicted by TargetScan for the miRNAs and genes which are experimentally validated to bind. Note that the effects of these SNPs on binding itself was not specifically evaluated experimentally for all cases. There are 108 SNP-miRNA pairs reported in this database for which the SNP lies in the miRNA target. We filtered out the duplicated pairs and polymorphisms longer than a single nucleotide. After filtering, we obtained 64 SNP-miRNA pairs for study. The results of evaluating the 64 pairs are given in Tables 2, 3, and 4.

As Table 2 shows, mrSNP reports the binding effects of 43 SNPs out of 64 (67\%) couples. For these miRNAmRNA couples, the SNPs either disrupt a match in the seed region or introduce a new $\mathrm{GU}$ wobble. For 19 of these pairs given in Table 2(a), the SNPs break a matching in the seed region, therefore, the minimum matching criteria cannot be satisfied. On the other hand, 4 of SNPs given at the top of Table 2(b) break a matching at the end of the seed region, resulting a $6 \mathrm{mer}$, for which the binding energies become lower than the threshold. Similarly, the other 20 SNPs in Table 2(b) introduce GU wobbles in the seed region, resulting to disturb the binding due to the minimum binding energy criteria.
Table 3 lists the 8 pairs for which mrSNP does not report a binding difference as the sequences for both alleles are predicted to bind the miRNAs at similar levels. Note that for the first two pairs in the Table 3, mrSNP captures the disruption in binding. However, mrSNP does not report these SNPs to affect binding, as it identifies another seed region for the miRNA in a location very close the original target. mrSNP identifies changes in the binding energies of the pairs in Table 3, which are ignored as the sequences for both alleles satisfy the minimum matching criteria. Table 4 lists another category of pairs that were not predicted by mrSNP. For these 13 pairs, the sequences for neither allele were calculated to bind the given miRNAs. The binding of these miRNAs are not predicted because the minimum matching criteria is not satisfied, as explained in more detail in the table.

We queried the 64 SNPs (Tables 2, 3, and 4) on mirSNP, PolymiRTS, and mirsnpscore. MirSNP reports 44 of these pairs as binding difference, and the results of MirSNP are very consistent with mrSNP. 57 of the 64 pairs queried are present in the PolymiRTS database, which includes miRNA-mRNA pairs identified through methods that include experimental data such as gene expression profiles and cross-linked immunoprecipitation sequencing data as well as pairs identified from the literature (rather than purely prediction methods). Because of the inclusion of experimental data and results from the literature, it is difficult to compare the results of PolymiRTS to mrSNP. Only 9 of the validated miRNA-mRNA pairs are found using mirsnpscore. Note that although the miRNAs-gene bindings are experimentally validated in this experiment, (Tables 2, 3, and 4), the actual effects of the SNPs on miRNA bindings are unknown. Therefore, it is not possible to determine the biological accuracy of the tools in this experiment. However, one result we can conclude from this experiment is that, mrSNP captures $51(43+8)$ of the $64(80 \%)$ experimentally validated miRNA bindings.

When comparing mirSNP to mrSNP across both experiments, 56 of 80 SNPs (70\%) were predicted by mirSNP to disrupt miRNA binding. mrSNP compares favorably by predicting that SNPs will disrupt the binding 54 of the 80 (68\%) miRNA target sites across these two experiments.

\section{Conclusion}

We developed a new tool, mrSNP, that predicts the effects of SNPs on miRNA binding. There are several advantages to this tool over existing tools. The proposed tool not only works on existing SNPs in databases such as dbSNP but also on novel SNPs which will be of great utility for researchers identifying new SNPs or somatic mutations in their samples. Secondly, our tool decreases the manual labor currently required for running prediction algorithms for novel SNPs. We present the results of mrSNP for various 3'UTR SNPs that were experimentally 
Table 2 SNP-miRNA pairs reported to disturb miRNA bindings by mrSNP for SNPs - miRNA couples obtained from miRdSNPs

\begin{tabular}{|c|c|c|c|c|c|c|c|}
\hline \multicolumn{8}{|c|}{ (a) Pairs captured with the minimum matching criteria } \\
\hline \multirow{2}{*}{$\frac{\text { SNP }}{\text { rs8829 }}$} & \multirow{2}{*}{$\begin{array}{l}\text { miRNA } \\
\text { hsa-miR-101 }\end{array}$} & \multirow{2}{*}{$\begin{array}{r}\text { Chr } \\
7\end{array}$} & \multirow{2}{*}{$\begin{array}{r}\text { Position } \\
148504618\end{array}$} & \multicolumn{2}{|c|}{ Alleles } & \multirow{2}{*}{$\frac{\text { Success }}{\checkmark}$} & \multirow{2}{*}{$\begin{array}{l}\text { Explanation } \\
\text { Binds one of the sequences with } 8 \text { consecutive matches } \\
\text { SNP breaks the match on } 2 \text { nd position, min. match critera } \\
\text { is not satisfied. }\end{array}$} \\
\hline & & & & C & A & & \\
\hline rs 28635788 & hsa-miR-124 & 2 & 47301624 & C & $\mathrm{T}$ & $\checkmark$ & $\begin{array}{l}\text { Binds one of the sequences with } 7 \text { consecutive matches. } \\
\text { SNP breaks the match on } 2 \text { nd position, min. match critera } \\
\text { is not satisfied. }\end{array}$ \\
\hline rs 28381252 & hsa-miR-224 & 19 & 45976504 & C & $\mathrm{T}$ & $\checkmark$ & $\begin{array}{l}\text { Binds one of the sequences with } 7 \text { consecutive matches. } \\
\text { SNP breaks the match on } 2 \text { nd position, min. match critera } \\
\text { is not satisfied. }\end{array}$ \\
\hline rs11782817 & hsa-miR-144 & 8 & 57074233 & A & C & $\checkmark$ & $\begin{array}{l}\text { Binds one of the sequences with } 10 \text { consecutive matches. } \\
\text { SNP breaks the match on } 2 \text { nd position, min. match critera } \\
\text { is not satisfied. }\end{array}$ \\
\hline rs11550076 & hsa-miR-25 & 9 & 110247352 & A & G & $\checkmark$ & $\begin{array}{l}\text { Binds one of the sequences with } 7 \text { consecutive matches. } \\
\text { SNP breaks the match on } 2 \text { nd position, min. match critera } \\
\text { is not satisfied. }\end{array}$ \\
\hline rs10196117 & hsa-miR-124 & 2 & 47301624 & C & $\mathrm{T}$ & $\checkmark$ & $\begin{array}{l}\text { Binds one of the sequences with } 7 \text { consecutive matches. } \\
\text { SNP breaks the match on } 2 \text { nd position, min. match critera } \\
\text { is not satisfied. }\end{array}$ \\
\hline rs1143552 & hsa-miR-181b & 22 & 33256174 & A & $G$ & $\checkmark$ & $\begin{array}{l}\text { Binds one of the sequences with } 8 \text { consecutive matches. } \\
\text { SNP breaks the match on the } 3 \text { rd position, min. match } \\
\text { critera is not satisfied. }\end{array}$ \\
\hline rs3733067 & hsa-miR-30a & 3 & 52290594 & A & G & $\checkmark$ & $\begin{array}{l}\text { Binds one of the sequences with } 7 \text { consecutive matches. } \\
\text { SNP breaks the match on } 4 \text { th position, min. match critera } \\
\text { is not satisfied. }\end{array}$ \\
\hline rs35180728 & hsa-miR-1 & 11 & 118473587 & - & $\mathrm{T}$ & $\checkmark$ & $\begin{array}{l}\text { Binds one of the sequences with } 7 \text { consecutive matches. } \\
\text { SNP breaks (deletes) the match on 4th position, min. } \\
\text { match critera is not satisfied. }\end{array}$ \\
\hline rs11557771 & hsa-miR-218 & 14 & 69341243 & A & C & $\checkmark$ & $\begin{array}{l}\text { Binds one of the sequences with } 8 \text { consecutive matches. } \\
\text { SNP breaks the match on } 6 \text { th position, min. match critera } \\
\text { is not satisfied. }\end{array}$ \\
\hline rs10055 & hsa-miR-30a & 16 & 24835876 & $\mathrm{~T}$ & C & $\checkmark$ & $\begin{array}{l}\text { Binds one of the sequences with } 8 \text { consecutive matches. } \\
\text { SNP breaks the match on } 6 \text { th position, min. match critera } \\
\text { is not satisfied. }\end{array}$ \\
\hline rs12635 & hsa-miR-197 & 19 & 14072442 & $C$ & $\mathrm{~T}$ & $\checkmark$ & $\begin{array}{l}\text { Binds one of the sequences with } 8 \text { consecutive matches. } \\
\text { SNP breaks the match on } 6 \text { th position, min. match critera } \\
\text { is not satisfied. }\end{array}$ \\
\hline rs3208684 & hsa-let-7g & 20 & 30252805 & $T$ & G & $\checkmark$ & $\begin{array}{l}\text { Binds one of the sequences with } 7 \text { consecutive matches. } \\
\text { SNP breaks the match on the } 6 \text { th position, min. match } \\
\text { critera is not satisfied. }\end{array}$ \\
\hline rs3208684 & hsa-let-7c & 20 & 30252805 & $\mathrm{~T}$ & G & $\checkmark$ & $\begin{array}{l}\text { Binds one of the sequences with } 7 \text { consecutive matches. } \\
\text { SNP breaks the match on the } 6 \text { th position, min. match } \\
\text { critera is not satisfied. }\end{array}$ \\
\hline rs5031032 & hsa-miR-1 & 12 & 102796132 & - & $\mathrm{T}$ & $\checkmark$ & $\begin{array}{l}\text { Binds one of the sequences with } 7 \text { consecutive matches. } \\
\text { SNP breaks (deletes) the match on 6th position, min. } \\
\text { match critera is not satisfied. }\end{array}$ \\
\hline rs1063320 & hsa-miR-148a & 6 & 29798749 & C & G & $\checkmark$ & $\begin{array}{l}\text { Binds one of the sequences with } 8 \text { consecutive matches. } \\
\text { SNP breaks the match on the } 7 \text { th position, min. match } \\
\text { critera is not satisfied. }\end{array}$ \\
\hline rs1063320 & hsa-miR-148b & 6 & 29798749 & C & G & $\checkmark$ & $\begin{array}{l}\text { Binds one of the sequences with } 7 \text { consecutive matches. } \\
\text { SNP breaks the match on the } 7 \text { th position, min. match } \\
\text { critera is not satisfied. }\end{array}$ \\
\hline rs1063320 & hsa-miR-152 & 6 & 29798749 & $C$ & G & $\checkmark$ & $\begin{array}{l}\text { Binds one of the sequences with } 7 \text { consecutive matches. } \\
\text { SNP breaks the match on the } 7 \text { th position, min. match } \\
\text { critera is not satisfied. }\end{array}$ \\
\hline
\end{tabular}


Table 2 SNP-miRNA pairs reported to disturb miRNA bindings by mrSNP for SNPs - miRNA couples obtained from miRdSNPs (Continued)

$\begin{array}{llllllll}\text { rs12831 hsa-miR-122 } \quad 16 \quad 30081561 \quad \text { A } & C & \checkmark & \begin{array}{l}\text { Binds one of the sequences with 8 consecutive matches. } \\ \text { SNP breaks the match on 7th position, min. match critera } \\ \text { is not satisfied. }\end{array}\end{array}$

\begin{tabular}{|c|c|c|c|c|c|c|c|}
\hline \multicolumn{8}{|c|}{ (b)Pairs captured with the minimum energy threshold } \\
\hline \multirow{2}{*}{$\frac{\text { SNP }}{\mathrm{rs} 9266}$} & \multirow{2}{*}{$\begin{array}{l}\text { miRNA } \\
\text { hsa-miR-181c }\end{array}$} & \multirow{2}{*}{$\begin{array}{r}\text { Chr } \\
12\end{array}$} & \multirow{2}{*}{$\begin{array}{r}\text { Position } \\
25362217\end{array}$} & \multicolumn{2}{|c|}{ Alleles } & \multirow{2}{*}{$\begin{array}{c}\text { Success } \\
\checkmark\end{array}$} & \multirow{2}{*}{$\begin{array}{l}\text { Explanation } \\
\text { Binds one of the sequences with } 7 \text { consecutive matches } \\
\text { SNP breaks the match on the 8th position (7th of seed) } \\
\text { the binding energy (of } 6 \text { mer) is lower than cut off. }\end{array}$} \\
\hline & & & & G & A & & \\
\hline 1434536 & hsa-miR-125b & 4 & 96075965 & $\mathrm{~T}$ & C & $\checkmark$ & $\begin{array}{l}\text { Binds one of the sequences with } 8 \text { consecutive matches. } \\
\text { SNP breaks the match on the 8th position ( } 7 \text { th of seed), } \\
\text { the binding energy (of } 6 \mathrm{mer} \text { ) is lower than cut off. }\end{array}$ \\
\hline rs17026326 & hsa-miR-19b & 3 & 30733356 & A & $\mathrm{T}$ & $\checkmark$ & $\begin{array}{l}\text { Binds one of the sequences with } 8 \text { consecutive matches. } \\
\text { SNP breaks the match on the 8th position ( } 7 \text { th of seed), } \\
\text { the binding energy (of } 6 \text { mer) is lower than cut off. }\end{array}$ \\
\hline rs17026326 & hsa-miR-19a & 3 & 30733356 & A & $\mathrm{T}$ & $\checkmark$ & $\begin{array}{l}\text { Binds one of the sequences with } 8 \text { consecutive matches. } \\
\text { SNP breaks the match on the 8th position ( } 7 \text { th of seed), } \\
\text { the binding energy (of } 6 \text { mer) is lower than cut off. }\end{array}$ \\
\hline rs3731562 & hsa-let-7d & 3 & 48199877 & G & A & $\checkmark$ & $\begin{array}{l}\text { Binds one of the sequences with } 8 \text { consecutive matches. } \\
\text { SNP introduces a GU wobble on } 2 \text { nd position, the binding } \\
\text { energy is lower than cut off. }\end{array}$ \\
\hline rs3731562 & hsa-let-7g & 3 & 48199877 & G & A & $\checkmark$ & $\begin{array}{l}\text { Binds one of the sequences with } 8 \text { consecutive matches. } \\
\text { SNP introduces a GU wobble on } 2 \text { nd position, the binding } \\
\text { energy is lower than cut off. }\end{array}$ \\
\hline
\end{tabular}

\begin{tabular}{lllllll}
\hline rs3731562 & hsa-let-7c & 3 & 48199877 G & A & $\checkmark$ & $\begin{array}{l}\text { Binds one of the sequences with 8 consecutive matches. } \\
\text { SNP introduces a GU wobble on 2nd position, the binding } \\
\text { energy is lower than cut off. }\end{array}$ \\
\hline
\end{tabular}

rs3731562 hsa-let-7b $\quad 3 \quad 48199877$ G $\quad$ A $\quad \checkmark \quad$ Binds one of the sequences with 8 consecutive matches.
SNP introduces a GU wobble on 2nd position, the binding energy is lower than cut off.

\begin{tabular}{|c|c|c|c|c|c|c|c|}
\hline rs3731562 & hsa-let-7a & 3 & 48199877 & G & A & $\checkmark$ & $\begin{array}{l}\text { Binds one of the sequences with } 8 \text { consecutive matches. } \\
\text { SNP introduces a GU wobble on } 2 \text { nd position, the binding } \\
\text { energy is lower than cut off. }\end{array}$ \\
\hline rs1051780 & hsa-miR-34a & 17 & 8063056 & C & $\mathrm{T}$ & $\checkmark$ & $\begin{array}{l}\text { Binds one of the sequences with } 7 \text { consecutive matches. } \\
\text { SNP introduces a GU wobble on 2nd position, the binding } \\
\text { energy is lower than cut off. }\end{array}$ \\
\hline rs59564714 & hsa-miR-15a & 11 & 73686038 & A & G & $\checkmark$ & $\begin{array}{l}\text { Binds one of the sequences with } 7 \text { consecutive matches. } \\
\text { SNP introduces a GU wobble on 3rd position, the binding } \\
\text { energy is lower than cut off. }\end{array}$ \\
\hline rs16952445/rs1138624 & hsa-miR-122 & 16 & 30081565 & C & $\mathrm{T}$ & $\checkmark$ & $\begin{array}{l}\text { Binds one of the sequences with } 7 \text { consecutive matches. } \\
\text { SNP introduces a GU wobble on 3rd position, the binding } \\
\text { energy is lower than cut off. }\end{array}$ \\
\hline rs1801938 & hsa-miR-1 & 19 & 2101071 & $\mathrm{~T}$ & G & $\checkmark$ & $\begin{array}{l}\text { Binds one of the sequences with } 7 \text { consecutive matches. } \\
\text { SNP introduces a GU wobble on } 3 \text { rd position, the binding } \\
\text { energy is lower than cut off. }\end{array}$ \\
\hline rs6875894 & hsa-miR-135b & 5 & 112179965 & C & $\mathrm{T}$ & $\checkmark$ & $\begin{array}{l}\text { Binds one of the sequences with } 9 \text { consecutive matches. } \\
\text { SNP introduces a GU wobble on 4th position, the binding } \\
\text { energy is lower than cut off. }\end{array}$ \\
\hline rs6875894 & hsa-miR-135a & 5 & 112179965 & C & $\mathrm{T}$ & $\checkmark$ & $\begin{array}{l}\text { Binds one of the sequences with } 9 \text { consecutive matches. } \\
\text { SNP introduces a GU wobble on 4th position, the binding } \\
\text { energy is lower than cut off. }\end{array}$ \\
\hline rs73306851 & hsa-miR-125b & 17 & 38327577 & A & G & $\checkmark$ & $\begin{array}{l}\text { Binds one of the sequences with } 7 \text { consecutive matches. } \\
\text { SNP introduces a GU wobble on } 5 \text { th position, the binding } \\
\text { energy is lower than cut off. }\end{array}$ \\
\hline rs11552766 & hsa-miR-185 & 3 & 49396652 & A & G & $\checkmark$ & $\begin{array}{l}\text { Binds one of the sequences with } 8 \text { consecutive matches. } \\
\text { SNP introduces a GU wobble on 5th position, the binding } \\
\text { energy is lower than cut off. }\end{array}$ \\
\hline
\end{tabular}


Table 2 SNP-miRNA pairs reported to disturb miRNA bindings by mrSNP for SNPs - miRNA couples obtained from miRdSNPs (Continued)

\begin{tabular}{llllllll}
\hline rs2664575 & hsa-miR-17 & 20 & 47862478 & $G$ & $T$ & $\checkmark$ & $\begin{array}{l}\text { Binds one of the sequences with 7 consecutive matches. } \\
\text { SNP breaks the match on the 6th position. }\end{array}$ \\
\hline rs10187 & hsa-miR-210 & 12 & 108962804 & $C$ & $T$ & $\checkmark$ & $\begin{array}{l}\text { Binds one of the sequences with } 7 \text { consecutive matches. } \\
\text { SNP introduces a GU wobble on 7th position, the binding } \\
\text { energy is lower than cut off. }\end{array}$
\end{tabular}

\begin{tabular}{|c|c|c|c|c|c|c|c|}
\hline midrule rs70965446 & hsa-miR-141 & 4 & 56301355 & A & G & $\checkmark$ & $\begin{array}{l}\text { Binds one of the sequences with } 9 \text { consecutive matches. } \\
\text { SNP introduces a GU wobble on } 7 \text { th position, the binding } \\
\text { energy is lower than cut off. }\end{array}$ \\
\hline rs8226 & hsa-miR-124 & 22 & 36677414 & G & $A$ & $\checkmark$ & $\begin{array}{l}\text { Binds one of the sequences with } 7 \text { consecutive matches. } \\
\text { SNP introduces a GU wobble on 7th position, the binding } \\
\text { energy is lower than cut off. }\end{array}$ \\
\hline rs35122558 & hsa-miR-155 & 7 & 32908099 & C & $\mathrm{T}$ & $\checkmark$ & $\begin{array}{l}\text { Binds one of the sequences with } 7 \text { consecutive matches. } \\
\text { SNP introduces a GU wobble on 8th position, the binding } \\
\text { energy is lower than cut off. }\end{array}$ \\
\hline rs55774542 & hsa-miR-125b & 12 & 48238167 & A & G & $\checkmark$ & $\begin{array}{l}\text { Binds one of the sequences with } 7 \text { consecutive matches. } \\
\text { SNP introduces a GU wobble on 8th position, the binding } \\
\text { energy is lower than cut off. }\end{array}$ \\
\hline rs59628511 & hsa-miR-124 & 9 & 140509654 & C & $\mathrm{G}$ & $\checkmark$ & $\begin{array}{l}\text { Binds one of the sequences with } 8 \text { consecutive matches. } \\
\text { SNP introduces a GU wobble on 8th position, the binding } \\
\text { energy is lower than cut off. }\end{array}$ \\
\hline
\end{tabular}

validated to disturb miRNA binding. We also compare the performance of mrSNP with other miRNA binding prediction tools, for which mrSNP performed better than all but one other platform, MirSNP, that had a success rate of $75 \%$ (Table 1). mrSNP correctly predicted 11 of $16(69 \%)$ disease-associated and/or experimentally validated SNPs that are reported in the literature or other databases. We observed that the recovery rate of mrSNP can be adjusted by using different set of parameters, but this may alter the false-positive rate. The major limitation of mrSNP is

Table 3 SNP-miRNA pairs predicted not to disturb the miRNA bindings by mrSNP on the SNPs - miRNA couples obtained from miRdSNP because both SNP alleles are predicted to bind the miRNAs

\begin{tabular}{|c|c|c|c|c|c|c|c|}
\hline SNP & miRNA & Chr & Position & & & Success & Explanation \\
\hline rs11556953 & hsa-miR-133a & 1 & 159888369 & A & $\mathrm{T}$ & $x$ & $\begin{array}{l}\text { Binds one of the sequences with } 7 \text { consecutive matches. } \\
\text { SNP breaks the match on } 2 \text { nd position. Binding difference } \\
\text { is not reported because miRNA still have another target } \\
\text { with } 8 \text { consecutive matches near the SNP location. }\end{array}$ \\
\hline
\end{tabular}

\begin{tabular}{|c|c|c|c|c|c|c|c|}
\hline rs13203 & hsa-miR-373 & 1 & 145442387 & $A$ & $C$ & $x$ & $\begin{array}{l}\text { Binds one of the sequences with } 7 \text { consecutive matches. } \\
\text { SNP breaks the match on 4th position. Binding difference } \\
\text { is not reported because miRNA still have another target } \\
\text { with } 7 \text { consecutive matches near the SNP location. }\end{array}$ \\
\hline rs3218074 & hsa-miR-424 & 19 & 30315176 & $A$ & G & $x$ & $\begin{array}{l}\text { miRNA is predicted to bind both of the sequences. SNP } \\
\text { is on the } 1 \text { st position, there are } 7 \text { consecutive matches in } \\
\text { both. }\end{array}$ \\
\hline rs36076633 & hsa-miR-1 & 1 & 159888513 & - & $G$ & $x$ & $\begin{array}{l}\text { miRNA is predicted to bind both of the sequences. SNP } \\
\text { is on the 1st position, there are } 7 \text { consecutive matches in } \\
\text { both. }\end{array}$ \\
\hline rs1059479 & hsa-miR-138 & 1 & 113243892 & A & $C$ & $\times$ & $\begin{array}{l}\text { miRNA is predicted to bind both of the sequences. SNP } \\
\text { is on the 1st position, there are } 8 \text { consecutive matches in } \\
\text { both. }\end{array}$ \\
\hline rs3218074 & hsa-miR-15b & 19 & 30315176 & $A$ & $G$ & $x$ & $\begin{array}{l}\text { miRNA is predicted to bind both of the sequences. SNP } \\
\text { introduces a GU wobble on } 1 \text { st position, there are } 8 \\
\text { consecutive matches in both. }\end{array}$ \\
\hline rs3218074 & hsa-miR-15a & 19 & 30315176 & $A$ & G & $\times$ & $\begin{array}{l}\text { miRNA is predicted to bind both of the sequences. SNP } \\
\text { introduces a GU wobble on 1st position, there are } 8 \\
\text { consecutive matches in both. }\end{array}$ \\
\hline rs3218074 & hsa-miR-16 & 19 & 30315176 & A & G & $\times$ & $\begin{array}{l}\text { miRNA is predicted to bind both of the sequences. SNP } \\
\text { introduces a GU wobble on 1st position, there are } 8 \\
\text { consecutive matches in both. }\end{array}$ \\
\hline
\end{tabular}


Table 4 SNP-miRNA pairs not predicted to disturb the miRNA bindings by mrSNP on the SNPs - miRNA couples obtained from miRdSNP because neither SNP allele is predicted to bind the miRNAs

\begin{tabular}{|c|c|c|c|c|c|c|c|}
\hline SNP & miRNA & Chr & Position & & & Success & Explanation \\
\hline rs16952475 & hsa-miR-185 & 15 & 69018917 & C & $\mathrm{T}$ & $\times$ & $\begin{array}{l}\text { miRNA is predicted not to bind neither of the sequences. } \\
\text { There are } 6 \text { consecutive matches, the binding energy is } \\
\text { lower than cut off. The SNP further introduces GU wobble } \\
\text { on } 5 \text { th position. }\end{array}$ \\
\hline rs17168525 & hsa-let-7b & 7 & 135613262 & $A$ & G & $\times$ & $\begin{array}{l}\text { miRNA is predicted not to bind neither of the sequences. } \\
\text { There are } 6 \text { consecutive matches, the binding energy is } \\
\text { lower than cut off. The SNP further introduces GU wobble } \\
\text { on } 5 \text { th position. }\end{array}$ \\
\hline rs1802677 & hsa-miR-181b & 22 & 33258866 & A & $\mathrm{T}$ & $x$ & $\begin{array}{l}\text { miRNA is predicted not to bind neither of the sequences. } \\
\text { There are } 6 \text { consecutive matches, the binding energy is } \\
\text { lower than cut off. The SNP further breaks the match on } \\
\text { 4th position. }\end{array}$ \\
\hline
\end{tabular}

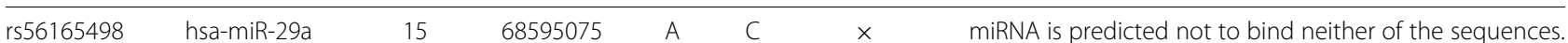
There are 6 consecutive matches, the binding energy is lower than cut off. The SNP further breaks the match on 5th position.

\begin{tabular}{|c|c|c|c|c|c|c|c|}
\hline rs57321187 & hsa-miR-192 & 10 & 79550563 & C & $\mathrm{T}$ & $\times$ & $\begin{array}{l}\text { miRNA is predicted not to bind neither of the sequences. } \\
\text { There are } 6 \text { consecutive matches, the binding energy is } \\
\text { lower than cut off. The SNP further breaks the match on } \\
\text { 6th position. }\end{array}$ \\
\hline rs62062994 & hsa-miR-29b & 17 & 48261978 & G & $\mathrm{T}$ & $\times$ & $\begin{array}{l}\text { miRNA is predicted not to bind neither of the sequences. } \\
\text { There are } 6 \text { consecutive matches, the binding energy is } \\
\text { lower than cut off. The SNP further breaks the match on } \\
\text { 3rd position. }\end{array}$ \\
\hline
\end{tabular}

rs62062994 hsa-miR-29c $\quad 17 \quad 48261978 \quad G \quad T \quad$ G $\quad$ miRNA is predicted not to bind neither of the sequences.
There are 6 consecutive matches, the binding energy is lower than cut off. The SNP further breaks the match on 3rd position.

\begin{tabular}{|c|c|c|c|c|c|c|c|}
\hline rs7233791 & hsa-miR-124 & 18 & 47309884 & C & $G$ & $x$ & $\begin{array}{l}\text { miRNA is predicted not to bind neither of the sequences. } \\
\text { There are } 6 \text { consecutive matches, the binding energy is } \\
\text { lower than cut off. The SNP further breaks the match on } \\
\text { 5th position. }\end{array}$ \\
\hline rs73954984 & hsa-miR-17 & 2 & 111925932 & C & $\mathrm{T}$ & $\times$ & $\begin{array}{l}\text { miRNA is predicted not to bind neither of the sequences. } \\
\text { There are } 6 \text { consecutive matches, the binding energy is } \\
\text { lower than cut off. The SNP further breaks the match on } \\
\text { 7th position. }\end{array}$ \\
\hline rs1804734 & hsa-miR-21 & 1 & 203278606 & $A$ & $G$ & $\times$ & $\begin{array}{l}\text { miRNA is predicted not to bind neither of the sequences. } \\
\text { There are } 7 \text { consecutive matches with a GU wobble, the } \\
\text { binding energy is lower than cut off. The SNP further } \\
\text { breaks the match on 6th position. }\end{array}$ \\
\hline rs3802782 & hsa-let-7b & 11 & 69468919 & $\mathrm{~T}$ & $C$ & $\times$ & $\begin{array}{l}\text { miRNA is predicted not to bind neither of the sequences. } \\
\text { There are } 7 \text { consecutive matches with a GU wobble, the } \\
\text { binding energy is lower than cut off in both. The SNP is on } \\
11 \text { th position, it further reduces the binding energy. }\end{array}$ \\
\hline rs3218074 & hsa-miR-503 & 19 & 30315176 & A & G & $\times$ & $\begin{array}{l}\text { miRNA is predicted not to bind neither of the sequences. } \\
\text { There are } 7 \text { consecutive matches with a GU wobble, the } \\
\text { binding energy is lower than cut off. The SNP further } \\
\text { introduces GU wobble on 1st position. }\end{array}$ \\
\hline rs1803045 & hsa-miR-1 & 12 & 49330252 & C & $\mathrm{T}$ & $x$ & $\begin{array}{l}\text { miRNA is predicted not to bind neither of the sequences. } \\
\text { There are } 6 \text { consecutive matches with a GU wobble, min. } \\
\text { match criteria cannot be satisfied in both. The SNP is on } \\
\text { 12th position, it further reduces the binding energy. }\end{array}$ \\
\hline
\end{tabular}

that it did not capture all of the SNPs experimentally predicted to disrupt miRNA binding. In future experiments, we will study additional larger sets of experimentally validated SNPs to improve the sensitivity and specificity of our binding predictions. As the literature is beginning to note miRNA binding to other regions of mRNAs and the potential for an influence on the 3'UTR location on binding, we will strive to incorporate these into our algorithms. 
In summary, mrSNP is a highly adaptable and performing tool for predicting the effect a SNP will have on miRNA binding.

\section{Availability and requirements}

Project name: mrSNP;

Project home page: http://mrsnp.osu.edu;

Operating system(s): Platform independent;

Programming language: Python, PHP and JavaScript;

Other requirements: JavaScript compatible browser;

License: Free for commercial and academic use;

Any restrictions to use by non-academics: No specific restrictions.

Competing interests

The authors declare that they have no competing interests.

\section{Authors' contributions}

AET and UVC conceived of the study, and participated in its design and coordination and helped to draft the manuscript. MD developed the workflow, ran the experiments, and wrote the manuscript. All authors read and approved the final manuscript.

\section{Acknowledgements}

This work was partially supported by NIH/NCI R01 CA134461.

\section{Author details}

'Biomedical Informatics, Computer Science and Engineering, The Ohio State University, Columbus, Ohio, USA. ${ }^{2}$ Biomedical Informatics, Electrical and Computer Engineering, The Ohio State University, Columbus, Ohio, USA. ${ }^{3}$ Molecular Virology, Immunology and Medical Genetics, The Ohio State University, Columbus, Ohio, USA.

Received: 27 August 2013 Accepted: 26 February 2014

Published: 15 March 2014

\section{References}

1. Friedman RC, Farh KK-H, Burge CB, Bartel DP: Most mammalian mrnas are conserved targets of micrornas. Genome Res 2009, 19(1):92-105.

2. Min $\mathrm{H}$, Yoon S: Got target? Computational methods for microRNA target prediction and their extension. Exp Mol Med 2010, 42:233-244

3. Lewis BP, Burge CB, Bartel DP: Conserved seed pairing, often flanked by adenosines, indicates that thousands of human genes are microrna targets. Cell 2005, 120(1):15-20.

4. Enright AJ, John B, Gaul U, Tuschl T, Sander C, Marks DS: MicroRNA targets in Drosophila. Genome Biol 2003, 5:1

5. Rehmsmeier M, Steffen P, Hochsmann M, Giegerich R: Fast and effective prediction of microRNA/target duplexes. RNA 2004, 10:1507-1517.

6. Rusinov V, Baev V, Minkov IN, Tabler M: Microlnspector: a web tool for detection of miRNA binding sites in an RNA sequence. Nucleic Acids Res 2005, 33:696-700.

7. Krek A, Grün D, Poy MN, Wolf R, Rosenberg L, Epstein EJ, MacMenamin P, da Piedade I, Gunsalus KC, Stoffel M, Rajewsky N: Combinatorial microrna target predictions. Nat Genet 2005, 37(5):495-500

8. Maragkakis M, Reczko M, Simossis VA, Alexiou P, Papadopoulos GL, Dalamagas T, Giannopoulos G, Goumas G, Koukis E, Kourtis K, Vergoulis T, Koziris N, Sellis T, Tsanakas P, Hatzigeorgiou AG: DIANA-microT web server: elucidating microRNA, functions through target prediction. Nucleic Acids Res 2009, 37:273-276.

9. Maragkakis M, Alexiou P, Papadopoulos GL, Reczko M, Dalamagas T, Giannopoulos G, Goumas G, Koukis E, Kourtis K, Simossis VA, Sethupathy P, Vergoulis T, Koziris N, Sellis T, Tsanakas P, Hatzigeorgiou AG: Accurate microrna target prediction correlates with protein repression levels. BMC Bioinformatics 2009, 295(1).

10. Sethupathy P, Collins FS: MicroRNA target site polymorphisms and human disease. Trends Genet 2008, 24:489-497.
11. Chen K, Rajewsky N: Natural selection on human microrna binding sites inferred from snp data. Nat Genet 2006, 38(12):1452-1456.

12. Chen K, Song F, Calin GA, Wei Q, Hao X, Zhang W: Polymorphisms in microrna targets: a gold mine for molecular epidemiology. Carcinogenesis 2008, 29(7):1306-1311.

13. Nicoloso MS, Sun H, Spizzo R, Kim H, Wickramasinghe P, Shimizu M, Wojcik SE, Ferdin J, Kunej T, Xiao L, Manoukian S, Secreto G, Ravagnani F, Wang X, Radice P, Croce CM, Davuluri RV, Calin GA: Single-nucleotide polymorphisms inside microrna target sites influence tumor susceptibility. Cancer Res 2010, 70(7):2789-2798.

14. Richardson K, Lai C-Q, Parnell L, Lee Y-C, Ordovas J: A genome-wide survey for snps altering microrna seed sites identifies functional candidates in gwas. BMC Genomics 2011, 12(1):504.

15. Greliche N, Zeller T, Wild PS, Rotival M, Schillert A, Ziegler A, Deloukas $P$, Erdmann J, Hengstenberg C, Ouwehand WH, Samani NJ, Schunkert H, Munzel T, Lackner KJ, Cambien F, Goodall AH, Tiret L, Blakenberg S, Tregouet D-A: Comprehensive exploration of the effects of mirna snps on monocyte gene expression. PloS one 2012, 7(9):45863.

16. Barenboim M, Zoltick BJ, Guo Y, Weinberger DR: MicroSNiPer: a web tool for prediction of SNP effects on putative microRNA targets. Hum Mutatition 2010, 31(11):1223-1232.

17. Hiard S, Charlier C, Coppieters W, Georges M, Baurain D: Patrocles: a database of polymorphic mirna-mediated gene regulation in vertebrates. Nucleic Acids Res 2010, 38(suppl 1):640-651.

18. Thomas LF, Saito T, Sætrom P: Inferring causative variants in microrna target sites. Nucleic Acids Res 2011, 39(16):109-109.

19. Bruno AE, Li L, Kalabus JL, Pan Y, Yu A, Hu Z: mirdsnp: a database of disease-associated snps and microrna target sites on $3^{\prime}$ utrs of human genes. BMC Genomics 2012, 13(1):44.

20. Liu C, Zhang F, Li T, Lu M, Wang L, Yue W, Zhang D: Mirsnp, a database of polymorphisms altering mirna target sites, identifies mirna-related snps in gwas snps and eqtls. BMC Genomics 2012, 13(1):661

21. Ziebarth JD, Bhattacharya A, Chen A, Cui Y: Polymirts database 2.0: linking polymorphisms in microrna target sites with human diseases and complex traits. Nucleic Acids Res 2012, 40(D1):216-221.

22. Kent WJ, Sugnet CW, Furey TS, Roskin KM, Pringle TH, Zahler AM, Haussler D: The human genome browser at UCSC. Genome Res 2002 12:996-1006.

23. Griffiths-Jones S, Saini HK, van Dongen S, Enright AJ: mirbase: tools for microrna genomics. Nucleic Acids Res 2008, 36(suppl 1):154-158.

doi:10.1186/1471-2105-15-73

Cite this article as: Deveci et al.: mrSNP: Software to detect SNP effects on microRNA binding. BMC Bioinformatics 2014 15:73.

Submit your next manuscript to BioMed Central and take full advantage of:

- Convenient online submission

- Thorough peer review

- No space constraints or color figure charges

- Immediate publication on acceptance

- Inclusion in PubMed, CAS, Scopus and Google Scholar

- Research which is freely available for redistribution 